\title{
Utilidad del monitoreo ambulatorio de la presión arterial de 24 horas en una población con alto riesgo cardiovascular
}

\author{
Usefulness of 24-hour ambulatory blood pressure monitoring in a population with high \\ cardiovascular risk
}

\author{
Orlando D. Navarro-Ulloa, Yelson A. Picón-Jaimes*, Giancarlos Conde-Cardona, \\ Luis J. Fernández-Yépez, Carmen I. Zabala-Carballo, Jonathan López-García, \\ Angélica M. Gómez-Hernández, Javier E. Orozco-Chinome y Luis R. Moscote-Salazar \\ Servicio de Cardiología, Instituto de Cardiología del Caribe S.A.S., Cartagena, Colombia
}

\section{Resumen}

Antecedentes: La hipertensión arterial es considerada una enfermedad y al mismo tiempo un factor de riesgo cardiovascular, involucrada principalmente en la cardiopatía isquémica, la enfermedad cerebrovascular y la insuficiencia renal, causando una elevada mortalidad. Objetivo: Realizar seguimiento con monitoreo ambulatorio de la presión arterial de 24 horas en pacientes con hipertensión arterial pertenecientes a una población con alto riesgo cardiovascular. Método: Estudio descriptivo, observacional, retrospectivo, en el que se analizaron los monitoreos ambulatorios de presión de 24 horas de 1858 pacientes, en Cartagena, Colombia. Resultados: Se incluyeron 1173 registros. La mediana de edad fue de 66 años. El 66.8\% (783) fueron mujeres y el 33.2\% (390) fueron hombres. Las principales alteraciones ocurrieron durante la noche, cuando el $79.1 \%$ de los pacientes tuvieron cargas elevadas de presión sistólica, el 65.6\% registraron promedios elevados de presión diastólica y el $83.7 \%$ tuvieron patrones circadianos anormales. Solo el 11\% de los estudios fueron normales en todos los parámetros. Conclusiones: El monitoreo ambulatorio de la presión arterial de 24 horas demostró ser una herramienta útil para identificar a los pacientes hipertensos no controlados, detectando hipertensión nocturna y patrones circadianos anormales, los cuales son marcadores de riesgo para morbilidad y mortalidad cardiovascular.

Palabras clave: Cardiología. Diagnóstico. Enfermedades cardiovasculares. Enfermedades vasculares. Hipertensión. Presión sanguínea.

\begin{abstract}
Background: High blood pressure is considered a disease and at the same time a cardiovascular risk factor, mainly involved in ischemic heart disease, cerebrovascular disease and kidney failure, causing high mortality worldwide. Objective: The objective was to follow up with 24-hour ambulatory blood pressure monitoring in patients with high blood pressure belonging to a population with high cardiovascular risk. Method: Descriptive, observational, retrospective study, which analyzes 24-hour outpatient pressure controls of 1858 patients, in Cartagena, Colombia. Results: 1173 exams were validated and included in the study. The median age was 66 years. $66.8 \%$ (783) were women and 33.2\% (390) were men. The main changes occurred
\end{abstract}

\section{Correspondencia:}

*Yelson A. Picón-Jaimes

Avda. Pocuro, 2260

Providencia

Fecha de recepción: 15-09-2019

C.P. 7500000 , Santiago, Chile

E-mail: ypicon@unab.edu.co

0009-7411/@ 2020 Academia Mexicana de Cirugía. Publicado por Permanyer. Este es un artículo open access bajo la licencia CC BY-NC-ND
Cir Cir. 2020;88(5):617-623

Contents available at PubMed

www.cirugiaycirujanos.com (http://creativecommons.org/licenses/by-nc-nd/4.0/). 
during the night, when $79.1 \%$ of the patients had high systolic pressure loads, $65.6 \%$ recorded diastolic pressure averages and $83.7 \%$ had abnormal circadian patterns. Only $11 \%$ of the studies were normal in all parameters. Conclusions: 24-hour ambulatory blood pressure monitoring proved to be a useful tool to identify uncontrolled hypertensive patients, detect nocturnal hypertension and abnormal circadian patterns, which are risk markers for cardiovascular morbidity and mortality.

Key words: Cardiology. Diagnosis. Cardiovascular diseases. Vascular diseases. Hypertension. Blood pressure.

\section{Introducción}

Las enfermedades cardiovasculares constituyen la primera causa de mortalidad en el mundo con 17.3 millones de muertes anuales y una tendencia hacia el aumento que se calcula llegará a una cifra de 23.6 millones en 20301. En el año 2013, la patología cardiovascular ocasionó el $31 \%$ de la mortalidad global, ocurriendo el $80 \%$ de esos fallecimientos en los países en vías de desarrollo'.

La prevalencia mundial de ataques cerebrovasculares en 2010 fue de 33 millones, con 16.9 millones de sujetos sufriendo su primer evento durante ese año, y en 2013 fueron la segunda causa de muerte, detrás de las enfermedades del corazón'. El $77 \%$ de las personas que presentan un primer accidente cerebrovascular tienen antecedente de hipertensión arterial, la cual es favorecida por estilos de vida poco saludables, especialmente en los países de bajos recursos económicos ${ }^{2}$.

Los factores de riesgo cardiovascular se clasifican en causales, mayores 0 independientes, al haberse demostrado una relación causal directa con las enfermedades cardiovasculares, y entre ellos están la hipertensión arterial, la diabetes mellitus, la hipercolesterolemia, el tabaquismo y la edad, explicando el $75 \%$ de los eventos fatales y no fatales ${ }^{3}$.

Otros factores de riesgo son los predisponentes y los condicionales. Los primeros actúan a través de otros factores de riesgo intermedios o guardando una clara asociación, y los segundos se ven durante el desarrollo de la enfermedad, pero sin evidencia de una relación causal directa ${ }^{3}$.

La hipertensión arterial es una enfermedad y también un factor de riesgo cardiovascular, siendo un principio causal para cardiopatía isquémica, insuficiencia cardiaca, enfermedad cerebrovascular, enfermedad arterial periférica, falla renal crónica, fibrilación auricular y mortalidad en todo el mundo ${ }^{4}$. Es la tercera causa de discapacidad inducida por enfermedad, siguiendo a la desnutrición y las enfermedades de transmisión sexual ${ }^{2}$. Se estima que la hipertensión afecta al $40 \%$ de la población mayor de 25 años ${ }^{5}$.
El control de los factores de riesgo mayores modificables (hipertensión, diabetes, hipercolesterolemia y tabaquismo) disminuye el riesgo de desarrollar enfermedades cardiovasculares (prevención primaria) y reduce el riesgo de nuevos eventos y de muertes prematuras (prevención secundaria) ${ }^{3}$.

También está comprobado que el control de la presión arterial reduce un $20 \%$ la mortalidad de causa coronaria y un $24 \%$ la mortalidad de causa cerebrovascular $^{3}$. Incluso se ha identificado a la hipertensión arterial nocturna como el mejor predictor de morbilidad y mortalidad cardiovascular, y su control está relacionado con una vida libre de eventos y de complicaciones cardiovasculares ${ }^{4-8}$.

Un metaanálisis de 17,312 pacientes hipertensos de tres continentes estableció la relación de los diferentes patrones circadianos non-dipper y riser (disminución inadecuada y aumento de la presión arterial durante el sueño) con la morbilidad y la mortalidad $^{7}$. Sin embargo, aunque existen estudios que demuestran que el control de la hipertensión mejora la calidad de vida de los pacientes, la media global de control con el tratamiento de la hipertensión solo es del $32.5 \%{ }^{5}$.

En Canadá, el 23\% de la población adulta es hipertensa, y hay que resaltar la mejoría en el control, que pasó del $13.2 \%$ en 1992 al $68.2 \%$ en 2012-2013. Entre los canadienses diabéticos, la prevalencia de la hipertensión arterial es del $67.1 \%$, y de ellos, el $60.1 \%$ están controlados ${ }^{5,9}$.

Un $33 \%$ de los adultos estadounidenses tienen hipertensión arterial, lo cual corresponde a 80 millones de personas, y a pesar de que el $77 \%$ están recibiendo tratamiento antihipertensivo solo el $54 \%$ de ellos están controlados y el $46 \%$ tienen la presión arterial por encima de 140/90 mmHg. Entre la población afroamericana, la hipertensión arterial es más frecuente y alcanza el $46 \%$, mientras que entre los hispanos (hombres y mujeres) es del 30\%.

En América Latina, un estudio que incluyó 11 países mostró que la prevalencia de la hipertensión arterial era del $40.7 \%$; de estos, el $40.6 \%$ estaban en tratamiento farmacológico, pero solo el $13.1 \%$ estaban 
controlados con unas cifras de presión arterial $<140 / 90 \mathrm{mmHg}^{2}$.

Cuatro países sudamericanos fueron incluidos en el estudio PURE: Argentina, Brasil, Colombia y Chile. De los pacientes hipertensos, el $57 \%$ tenían conocimiento de su condición, pero de ellos solo el $52.8 \%$ recibían tratamiento y únicamente el $18.3 \%$ estaban controlados $^{2,10}$.

En Colombia, la prevalencia de la hipertensión arterial evidenciada en una revisión sistemática realizada en 2014 fue del $31.33 \%$ (25.95\% en 2010$)$, con una tasa de mortalidad del $12.71 \%$ ( $13.88 \%$ en 2010 ), en las mujeres, y una prevalencia del $28.73 \%$ ( $23.85 \%$ en 2010), con una tasa de mortalidad del $13.73 \%$ (14.81\% en 2010), en los hombres ${ }^{11}$.

El monitoreo ambulatorio de la presión arterial ha demostrado ser una herramienta útil para el diagnóstico y el seguimiento de la enfermedad. Los pacientes con hipertensión «de bata blanca», hipertensión arterial enmascarada, resistente y pseudorresistente pueden ser identificados con este método diagnóstico ${ }^{12}$. Un estudio transversal multicéntrico realizado en unidades de atención primaria de la Comunidad Valenciana (España), que incluyó 1028 pacientes hipertensos mayores de 65 años, demostró que el monitoreo ambulatorio de la presión arterial fue superior a las mediciones clínicas para identificar a los pacientes controlados ${ }^{13,14}$.

Se ha demostrado que las mediciones de la presión arterial con monitoreo ambulatorio guardan una mayor relación con el daño a órganos diana, y que la presión de pulso elevada durante el sueño, el comportamiento circadiano anormal de la presión arterial, y en especial el promedio de la presión arterial nocturna, son predictores de morbilidad y mortalidad de causa cardiovascular ${ }^{15}$.

Por lo tanto, es perentorio determinar esos parámetros mediante el monitoreo ambulatorio de la presión arterial en todos los pacientes hipertensos, y más si tienen alto riesgo cardiovascular, para realizar los ajustes necesarios en el tratamiento antihipertensivo que les generen un beneficio neto reflejado en una vida más larga y de mejor calidad.

El objetivo de este estudio fue realizar un seguimiento con monitoreo ambulatorio de la presión arterial de 24 horas en pacientes con hipertensión arterial pertenecientes a una población con alto riesgo cardiovascular.

\section{Método}

Estudio descriptivo, observacional, transversal y retrospectivo, realizado en 1858 pacientes del programa de alto riesgo cardiovascular remitidos a cardiodinamia desde el 29 de septiembre de 2014 hasta el 31 de diciembre de 2015, quienes fueron relacionados y detallados en una base de datos calificados por sus médicos de prevención cardiovascular como pacientes de riesgo alto con eventos cardiovasculares previos, como infarto de miocardio, revascularización coronaria, accidente cerebrovascular, falla cardiaca, enfermedad renal crónica o diabetes mellitus.

A los pacientes se les realizó monitoreo ambulatorio de la presión arterial de 24 horas para detectar a aquellos con unas cifras por encima de los siguientes umbrales: $130 / 85 \mathrm{mmHg}$ promedio para las 24 horas, $135 / 90 \mathrm{mmHg}$ para el periodo diurno o de vigilia, $120 / 70 \mathrm{mmHg}$ para la noche o sueño, $25 \%$ para las cargas o porcentajes de presiones sistólica y diastólica por encima de los umbrales, y $50 \mathrm{mmHg}$ para la presión de pulso. Las presiones arteriales sistólica y diastólica deben disminuir durante el sueño un 10$20 \%$ con relación al promedio diurno. Los pacientes con ciclos circadianos normales se denominaron con patrón o perfil dipper, quienes tuvieron una disminución atenuada $(<10 \%)$ se denominaron non-dipper, y aquellos con una disminución $>20 \%$ se calificaron como extreme dipper. Cualquier aumento de las presiones durante el sueño o ciclo circadiano invertido se calificó como riser.

Todos los estudios de monitoreo ambulatorio de la presión arterial se realizaron con monitores recién adquiridos, marca Bravo de SunTech Medical, con AccuWinProSE ABPM Software, que cuenta con las validaciones ANSI/AAMI SP10:2002.

Los criterios de inclusión fueron: a) pacientes del programa de alto riesgo cardiovascular; b) monitoreo de la presión arterial de ingreso; y c) solo los estudios que cumplieran los criterios de validación de la Canadian Cardiovascular Society, entre los que están el registro mínimo por 24 horas, medidas cada $20-30$ minutos durante el día y cada 30-60 minutos durante la noche, siendo los criterios de éxito al menos un $70 \%$ de las mediciones válidas, al menos 20 medidas durante el día y al menos 7 medidas durante la noche. Teniendo en cuenta el horario para acostarse y levantarse de la mayoría de los pacientes de la zona geográfica y el estrato socioeconómico, los periodos fueron ajustados por defecto para el día o vigilia de 05:00 horas a 21:00 horas, y para la noche o sueño de las 21:00 horas hasta las 05:00 horas del día siguiente. A algunos pacientes se les personalizó el horario de los periodos.

Los criterios de exclusión fueron: a) pacientes no inscritos en el programa de alto riesgo cardiovascular; 
Tabla 1. Mediciones de los monitoreos ambulatorios de presión de $\mathbf{2 4}$ horas

\begin{tabular}{|c|c|c|c|c|c|c|c|}
\hline \multirow[t]{2}{*}{ Variable } & \multicolumn{2}{|c|}{24 horas } & \multicolumn{2}{|c|}{ Día } & \multicolumn{2}{|c|}{ Noche } & \multirow{2}{*}{$\frac{\text { Diferencia noche-día }}{\text { Mediana [RI] }}$} \\
\hline & Mediana [RI] & $\begin{array}{c}\text { Aumento* } \\
n(\%)\end{array}$ & Mediana [RI] & $\begin{array}{c}\text { Aumento* } \\
\mathrm{n}(\%)\end{array}$ & Mediana [RI] & $\begin{array}{c}\text { Aumento* } \\
\mathrm{n}(\%)\end{array}$ & \\
\hline Frecuencia cardiaca (I.p.m.) & $72[65-80]$ & $59(5.0)$ & - & - & - & - & - \\
\hline Presión de pulso (mmHg) & $56[49-76]$ & $823(70.2)$ & $56[49-66]$ & $810(69.0)$ & 57 [49-68] & $821(70.0)$ & $1[-3-6]$ \\
\hline PAS (mmHg) & 130 [118-142] & $602(51.3)$ & $130[118-144]$ & $453(38.6)$ & $128[115-143]$ & $770(65.6)$ & $-2[-9-5]$ \\
\hline Carga PAS (\%) & 38 [14-69] & $734(62.6)$ & 24 [6-58] & $584(49.8)$ & 75 [33-100] & $928(79.1)$ & $-1.6[-7.2-4.1]$ \\
\hline PAD $(\mathrm{mmHg})$ & $71[64-80]$ & $184(15.7)$ & $72[65-81]$ & $107(9.1)$ & 69 [62-77] & $538(45.9)$ & $-3[-8-1]$ \\
\hline Carga PAD (\%) & $11[2-30]$ & $357(30.4)$ & $6[0-24]$ & $290(24.7)$ & 20 [0-55] & $544(46.4)$ & $-4.5[-10.6-1.2]$ \\
\hline
\end{tabular}

b) estudios repetidos; y c) estudios que no cumplieran los requisitos de validación.

La fibrilación auricular permanente, los pulsos braquiales demasiado débiles o imperceptibles, la demencia senil o los trastornos cognitivos graves, y la enfermedad de Parkinson grave o con movimientos anormales 0 discinesias, fueron los principales motivos que impidieron realizar el monitoreo de presión arterial de 24 horas o para no completarlo.

Los datos de los estudios que sí cumplieron los criterios fueron cargados en el software MS Office Excel 2013. El análisis estadístico se realizó mediante el software Epi Info versión 7.2.

Este proyecto se elaboró de acuerdo con la resolución 008430 del Ministerio de Protección Social de Colombia, por el cual se regula la investigación en seres humanos, constituyendo un estudio sin riesgo, ya que los métodos utilizados no modifican ni alteran las variables biológicas, psicológicas ni sociales de los pacientes, lo cual es acorde con el Tratado de Helsinki y con las pautas CIOMS (Council for International Organizations of Medical Sciences) para investigación en seres humanos.

En todos los casos se obtuvo el consentimiento informado de los pacientes y se garantizó el resguardo de los datos de identificación.

\section{Resultados}

El programa de alto riesgo cardiovascular incluyó 1858 pacientes, pero tras su revisión inicial se consideraron 1352 casos que cumplían con el requisito de tener el monitoreo ambulatorio en 24 horas, de los cuales se excluyeron 179 por datos errados en los registros de la presión arterial diastólica en 24 horas, errores en el registro de la presión sistólica diurna o duplicación. Finalmente, se analizaron 1173 monitoreos de presión en 24 horas que cumplieron los requisitos de inclusión.

El promedio de edad fue de 66 años, con un rango intercuartílico de 56 a 76 años y una desviación estándar de \pm 13.7 años. En cuanto a la distribución por sexo, el $66.8 \%(n=783)$ eran mujeres y el $33.2 \%$ $(n=390)$ eran hombres.

El análisis de los resultados evidenció una mediana de frecuencia cardiaca de 72 latidos por minuto, presión de pulso de $56 \mathrm{mmHg}$, presión arterial sistólica de $130 \mathrm{mmHg}$ con carga del $38 \%$ y promedio de presión diastólica de $71 \mathrm{mmHg}$ con carga del 11\%. En cuanto a los periodos del día y la noche, se encontraron respectivamente unas medianas para la presión de pulso de 56 y $57 \mathrm{mmHg}$, para la presión sistólica de 130 y $128 \mathrm{mmHg}$ con cargas del $24 \%$ y el $75 \%$, y para la presión diastólica de 72 y $69 \mathrm{mmHg}$ con cargas del $5 \%$ y el $20 \%$. Durante el periodo de 24 horas se registró elevación de la presión de pulso en el $70.2 \%$ de los pacientes, hipertensión sistólica en el 51.3\% e hipertensión diastólica en el 15.7\%. La discriminación detallada de los hallazgos en los monitoreos de presión de 24 horas se muestra en la tabla 1.

El porcentaje de pacientes con elevación de la presión de pulso, aumento de la presión arterial sistólica y la presión arterial diastólica, así como el porcentaje de sujetos con aumento en las cargas sistólicas y diastólicas, se esquematiza en la figura 1.

Finalmente, al evaluar los patrones circadianos de las presiones arteriales sistólica y diastólica los resultados fueron: para la presión sistólica, el patrón 


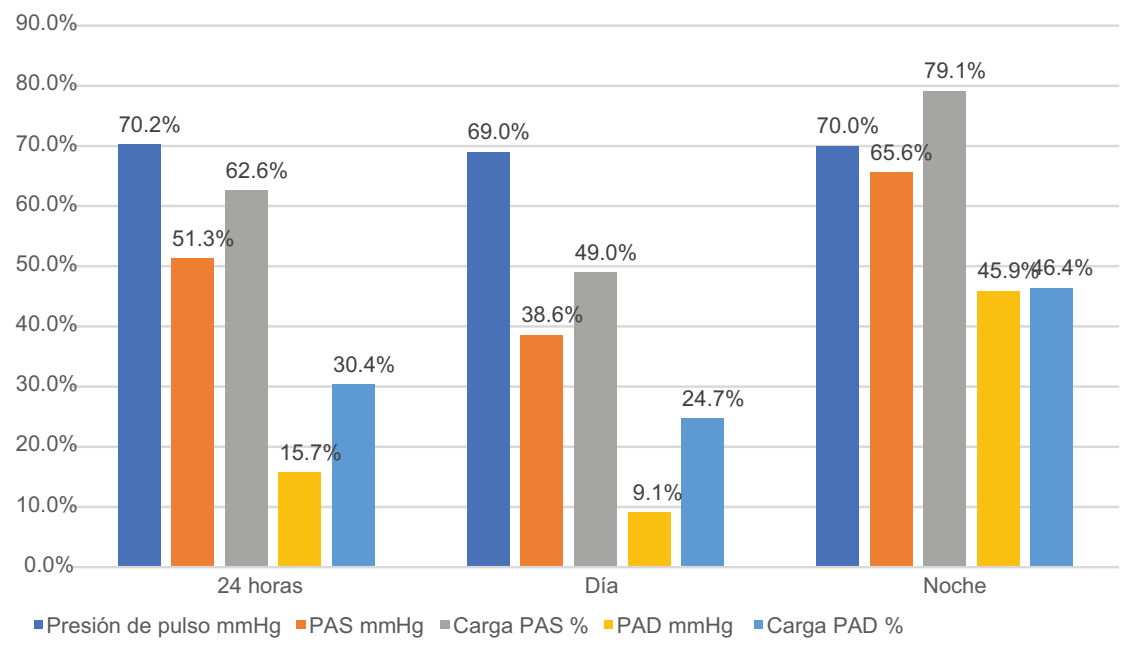

Figura 1. Porcentaje de pacientes con elevación de la presión de pulso, aumento de la presión arterial sistólica y la presión arterial diastólica, y cargas de presión. PAD: presión arterial diastólica; PAS: presión arterial sistólica.

non-dipper se observó en el $43.2 \%$ de los pacientes $(n=507)$, el riser en el $40.5 \%(n=475)$, solo el $15.6 \%$ de los pacientes $(n=183$ ) tuvieron un patrón normal o dipper, y el $0.7 \%(n=8)$ fueron extreme dipper. Así, el $84 \%$ de los pacientes tuvieron patrones circadianos anormales.

Los perfiles circadianos para la presión arterial diastólica fueron: patrón non-dipper en el $47.6 \%$ de los pacientes ( $n=558)$, riser en el $28.6 \%(n=336)$, perfil normal o dipper en el $22.2 \%(n=260)$ y extreme dipper en el $1.7 \%(n=20)$.

\section{Discusión}

En este grupo de pacientes clasificados con riesgo cardiovascular alto provenientes de un programa de control de riesgo cardiovascular que estaban recibiendo tratamiento antihipertensivo, el monitoreo ambulatorio de la presión arterial evidenció un control inadecuado en el $79.1 \%$. La carga de presión arterial sistólica durante la noche fue el marcador más alterado, seguida por la presión de pulso en el $70 \%$ durante la noche y la hipertensión sistólica nocturna en el $65.6 \%$. Adicionalmente, el $83.7 \%$ de los pacientes $(\mathrm{n}=982)$ registraron patrones circadianos anormales de la presión sistólica, siendo non-dipper el $43.2 \%$ $(n=507)$ y riser el $40.5 \%(n=475)$.

Solo el $16.3 \%$ de los pacientes pudieron considerarse controlados, lo cual es inaceptable para los demás, por su condición de riesgo cardiovascular alto y por contar con múltiples factores de riesgo cardiovascular asociados a la hipertensión arterial, como diabetes mellitus y dislipidemias o haber tenido eventos cardiovasculares o cerebrovasculares, falla cardiaca o enfermedad renal.

La relación entre la hipertensión arterial nocturna y los eventos cardiovasculares en una cohorte de 2115 pacientes hipertensos tratados con riesgo alto quedó establecida al demostrarse que la presión arterial sistólica $>130 \mathrm{mmHg}$ incrementó el riesgo de infartos no fatales, accidentes cerebrovasculares, hospitalización por falla cardiaca y muerte cardiovascular en el $52 \%$, en comparación con los que presentaban valores de presión arterial sistólica $<115 \mathrm{mmHg}^{8}$.

En varias publicaciones se ha establecido que el promedio de la presión arterial durante el periodo de noche o sueño y los patrones riser y non-dipper están directamente relacionados con la mortalidad y los eventos cardiovasculares ${ }^{4-8}$. En este estudio observamos que el $65.6 \%$ de los pacientes registraron hipertensión arterial nocturna, el $79.1 \%$ tuvieron cargas elevadas de presión arterial sistólica, el $40.5 \%$ mostraron un patrón circadiano riser y el $43.2 \%$ tuvieron un patrón non-dipper.

Los estudios han encontrado que la hipertensión arterial nocturna está relacionada con lesión subclínica a órganos diana, como aumento de la masa ventricular y engrosamiento de la capa íntima-media carotídea, e incluso algunos autores han informado de deterioro cognitivo en esos pacientes ${ }^{15-17}$.

En 2015, Androulakis, et al. ${ }^{16}$ compararon la presión arterial sistólica central con las presiones del monitoreo ambulatorio de presión arterial para pronosticar el daño subclínico a órganos diana en caso de 
hipertensión arterial nocturna, determinado por el cociente de proteinuria en orina y la disfunción mecánica del ventrículo izquierdo. Los resultados de su estudio indican que la media de la presión arterial nocturna mostró una correlación significativa con la presión arterial sistólica central y periférica, y se asoció significativamente con el aumento de la proteinuria. Los autores concluyen que la presión arterial sistólica nocturna se correlaciona mejor con la función mecánica del ventrículo izquierdo y la relación albuminuria/ creatinuria que la presión arterial sistólica central.

El monitoreo ambulatorio de la presión arterial es uno de los estudios cardiológicos más recientes y cada día se consolida más como una herramienta útil y eficiente, por su relación costo-beneficio, para mejorar el diagnóstico y el seguimiento de los pacientes hipertensos, optimizar el tratamiento antihipertensivo y establecer el pronóstico individual. Es también de utilidad ante la sospecha de hipertensión enmascarada en presencia de presión arterial normal en el consultorio, pero con datos de daño a órganos diana, siendo de primordial importancia en las mujeres, debido a que la relación entre eventos cardiovasculares o renales e hipertensión arterial nocturna es más frecuente en ellas, y si están embarazadas contribuye a precisar el riesgo maternofetal|18-20. En pacientes que requieren esquemas antihipertensivos complejos ayuda a ajustar el horario y las dosis de los medicamentos, reflejando en forma clara la función autonómica del paciente, la cual está estrechamente ligada al pronóstico y a la vida libre de eventos cardiovasculares ${ }^{21}$.

Estamos entonces ante la premisa de realizar un monitoreo ambulatorio de la presión arterial de 24 horas a todos los pacientes hipertensos, independientemente de su edad y de su comorbilidad, para garantizar el control de la presión arterial en forma completa y efectiva. Tener hipertensión arterial nocturna o un patrón circadiano anormal puede corregirse trasladando al horario de la noche algunos de los medicamentos antihipertensivos diferentes de los diuréticos, pero sin olvidar la posibilidad de patologías concurrentes, como apnea obstructiva del sueño, trastornos diversos del sueño, neuropatía/disautonomía diabética, etc.

En este grupo de pacientes de alto riesgo cardiovascular, el monitoreo ambulatorio de la presión arterial ha demostrado ser una herramienta muy útil para identificar a los hipertensos no controlados, en especial a aquellos con hipertensión arterial nocturna, patrones circadianos anormales o presión de pulso elevada.

La importancia de la información que brinda este estudio y los beneficios que se derivan del control adecuado de la hipertensión arterial, tales como reducir la mortalidad cardiovascular, revertir las alteraciones cognitivas que afectan la calidad de vida de los pacientes y evitar o detener la progresión de la falla cardiaca o de la falla renal, convierten al monitoreo ambulatorio de la presión arterial en imprescindible para el manejo de los pacientes hipertensos, y más aún si son de alto riesgo cardiovascular. Además del beneficio social, el beneficio económico para el sistema de salud sería muy importante, no solo en el valor de los medicamentos, sino también en el ahorro de días de incapacidad laboral, de consultas a los servicios de emergencia y de estancias hospitalarias.

En la época de la «polipíldora», los medicamentos antihipertensivos combinados y la tendencia a ordenar tomar todas las medicinas al levantarse, será necesario instruir a los pacientes con hipertensión arterial nocturna para que no permitan que, sin justificación, les modifiquen el esquema de tratamiento y el cronograma que les controló dicha alteración.

Como médicos, debemos ofrecer mejor información a los pacientes en general y en especial a los hipertensos, para motivarles a llevar un estilo de vida saludable incrementando la actividad física, el ejercicio aeróbico, la alimentación variada y balanceada, tomar más agua pura y prescindir de las bebidas azucaradas, mejorar su vida social, familiar y espiritual, y todo ello encaminado a mejorar su ecosistema interno y gozar de buena salud y felicidad.

\section{Conclusiones}

Es importante aclarar que para lograr un buen control de la hipertensión arterial se debe hacer seguimiento a los pacientes, preferiblemente con monitoreo ambulatorio de la presión arterial de 24 horas, pues se ha evidenciado que a pesar de recibir medicación por ser considerados pacientes de alto riesgo cardiovascular, el porcentaje con patrones anormales reportados en el monitoreo es muy elevado, lo cual continúa siendo un factor de riesgo para desenlaces fatales.

Una de las prioridades en el control de las cifras de presión arterial debe ser el control nocturno, pues la literatura médica mundial ha sido enfática en que no lograrlo se asocia a mayor mortalidad de causa cardiovascular.

Nos atrevemos a invitar a los lectores a realizar controles periódicos en el seguimiento de los pacientes hipertensos con comorbilidad mediante el monitoreo ambulatorio de la presión arterial de 24 horas, para tener una valoración real del comportamiento de la 
patología y tomar las medidas necesarias con el objetivo de disminuir la aparición de eventos cardiovasculares graves que puedan condicionar y limitar la funcionabilidad del paciente.

\section{Agradecimientos}

Un agradecimiento especial a Mary Vergara de Navarro, María Carolina Sánchez, Ramón Fortich, Juan Camilo Ulloa y Enrique Ramos.

\section{Conflictos de intereses}

Los autores manifiestan no tener conflictos de intereses.

\section{Responsabilidades éticas}

Protección de personas y animales. Los autores declaran que los procedimientos seguidos se conformaron a las normas éticas del comité de experimentación humana responsable y de acuerdo con la Asociación Médica Mundial y la Declaración de Helsinki.

Confidencialidad de los datos. Los autores declaran que han seguido los protocolos de su centro de trabajo sobre la publicación de datos de pacientes.

Derecho a la privacidad y consentimiento informado. Los autores declaran que en este artículo no aparecen datos de pacientes.

\section{Bibliografía}

1. Writing Group Members, Mozaffarian D, Benjamin EJ, Go AS, Arnett DK, Blaha MJ, Cushman M, et al. Heart disease and stroke statistics - 2016 update: a report from the American Heart Association. Circulation. 2016;133:e38-360.

2. López-Jaramillo P, Sánchez RA, Diaz M, Cobos L, Bryce A, Parra Carrillo JZ, et al.; Latin America Expert Group. Latin American consensus on hypertension in patients with diabetes type 2 and metabolic syndrome. J Hypertens. 2013;31:223-38.

3. Lobos JM, Brotons C. Factores de riesgo cardiovascular y atención primaria: evaluación e intervención. Aten Primaria. 2011;43:668-77.

4. Piepoli MF, Hoes AW, Agewall S, Albus C, Brotons C, Catapano AL, et al. 2016 European Guidelines on cardiovascular disease prevention in clinical practice: The Sixth Joint Task Force of the European Society of Cardiology and Other Societies on Cardiovascular Disease Prevention in Clinical Practice (Constituted by Representatives of 10 Societies and by Invited experts) Developed With the Special Contribution of the European Association for Cardiovascular Prevention \& Rehabilitation (EACPR). Eur Heart J. 2016;37:2315-81.
5. Leung AA, Nerenberg K, Daskalopoulou SS, McBrien K, Zarnke KB, Dasgupta K, et al. Hypertension Canada's 2016 Canadian Hypertension Education Program Guidelines for Blood Pressure Measurement, Diagnosis, Assessment of Risk, Prevention, and Treatment of Hypertension. Can J Cardiol. 2016:32:569-88.

6. Hermida RC, Ayala DE, Mojón A, Fernández JR. Decreasing sleep-time blood pressure determined by ambulatory monitoring reduces cardiovascular risk (the MAPEC Study). Am Coll Cardiol. 2011; 58:1165-73.

7. Salles GF, Reboldi G, Fagard RH, Cardoso CRL, Pierdomenico SD, Verdecchia $\mathrm{P}$, et al.; ABC-H Investigators. Prognostic effect of the nocturnal blood pressure fall in hypertensive patients. The Ambulatory Blood Pressure Collaboration in Patients With Hypertension $(\mathrm{ABC}-\mathrm{H})$ meta-analysis. Hypertension. 2016;67:693-700.

8. De la Sierra A, Banegas JR, Segura J, Gorostidi M, Ruilope LM. Ambulatory blood pressure monitoring and development of cardiovascular events in high-risk patients included in the Spanish ABPM registry: the CARDIORISC Event study. J Hypertens. 2012;30:713-9.

9. Padwal RS, Bienek A, McAlister FA, Campbell NRC; the Outcomes Research Task Force of the Canadian Hypertension Education Program. Epidemiology of hypertension in Canada: an update. Can $\mathrm{J}$ Cardiol. 2016:32:687-94.

10. Chow CK, Teo KK, Rangarajan S, Islam S, Gupta R, Avezum A, et al.; PURE (Prospective Urban Rural Epidemiology) Study investigators. Prevalence, awareness, treatment, and control of hypertension in rural and urban communities in high-, middle-, and low-income countries. JAMA. 2013;310:959-68.

11. Vargas-Sandoval G, Valencia-Hernández C, Castañeda-Orjuela C. Estimación de la carga de enfermedad por hipertensión arterial en Colombia. 2010-2014. En: Instituto Nacional de Salud, Observatorio Nacional de Salud, Quinto Informe ONS: carga de enfermedad por enfermedades crónicas no transmisibles y discapacidad en Colombia. Bogotá, D.C.: Imprenta Nacional de Colombia; 2015. p. 100-8.

12. Lehmann MV, Zeymer U, Dechend R, Kaiser E, Hagedorn I, Deeg E, et al. Ambulatory blood pressure monitoring: is it mandatory for blood pressure control in treated hypertensive patients? Prospective observational study. Int J Cardiol. 2013;168:2255-63.

13. O'Brien E, Parati G, Stergiou G, Asmar R, Beilin L, Bilo G, et al.; European Society of Hypertension Working Group on Blood Pressure Monitoring. European Society of Hypertension position paper on ambulatory blood pressure monitoring. J Hypertens. 2013;31:1731-68. [Erratum: J Hypertens. 2013;31:2467.]

14. Sobrino J, Domenech $M$, Camafort $M$, Vinyoles $E$, Coca A; investigadores del grupo ESTHEN. Prevalencia de hipertensión arterial enmascarada en una cohorte de pacientes hipertensos controlados en España. Med Clin (Barc). 2011;136:607-12

15. Turner JR, Viera AJ, Shimbo D. Ambulatory blood pressure monitoring in clinical practice: a review. Am J Med. 2015;128:14-20.

16. Androulakis E, Papageorgiou N, Chatzistamatiou E, Papaioannou S, Synetos A, Latsios G, et al. Non-dipping versus nocturnal hypertension: improving detection of preclinical organ damage in hypertension. JACC. 2015;65(10S)

17. Sierra C, Salamero M, Domenech M, Camafort M, Coca A. Circadian blood pressure pattern and cognitive function in middle-aged essential hypertensive patients. Rev Esp Cardiol. 2015;68:151-62.

18. Kim D, Shim CY, Hong G, Cho IJ, Chang HJ, Ha JW, et al. Implication of night-time mean systolic blood pressure for predicting subclinical target organ damage in hypertension: comparison with office central blood pressures. JACC 2016;67(Supl).

19. Duque M, Díaz JC, Molina DI, Gómez E, Márquez G, López $P$, et al. Neuropatía autonómica diabética cardiovascular. Rev Colomb Cardiol. 2013;20:80-7.

20. Bombardieri E, Giammarile F, Aktolun C, Baum RP, Delaloye AB, Maffioli $\mathrm{L}$, et al. 131l/123l-metaiodobenzylguanidine (mIBG) scintigraphy: procedure guidelines for tumour imaging. Eur J Nucl Med Mol Imaging. 2010;37:2436-46.

21. Minamisawa M, Motoki H, Izawa A, Kashima $\mathrm{Y}$, Miura T, Ebisawa S, et al. Incremental prognostic value of ambulatory blood pressure monitoring for cardiovascular events in patients with acute myocardial infarction: ALPS AMI subanalysis. JACC 2014;63(12). 